\title{
Assessment of hospitalization and mortality of scleroderma in-patients: a thirteen-year study
}

\author{
Saeedeh Shenavandeh, Razieh Naseri \\ Division of Rheumatology, Departments of Internal Medicine, Shiraz University of Medical Sciences, Iran
}

\begin{abstract}
Objective: Systemic sclerosis (SSc) is an uncommon non-hereditary sporadic disease that increases the risk of premature death, especially in diffuse type. We determined the prevalence of SSc in the last 13 years in our rheumatologic hospitals as a referral center for southern Iranian patients, the causes of hospitalization, the average length of stay (LOS), the mortality rate, and the reason for their mortality.

Material and methods: A cross-sectional study was performed in Shiraz University of Medical Sciences, Iran. The studied population included all patients diagnosed with systemic sclerosis. We calculated the hospitalization rates, in-hospital mortality rates, and mean LOS.

Results: There were 446 admissions by 181 patients with SSc. The female to male ratio was about 10.7 : 1. The overall mean LOS was 5.95 days. Digital ulcer and interstitial lung disease (ILD) were the most common causes of hospitalizations among the SSc-related events. For those with a non-SSc-related condition, infection was the most prevalent event. Most of the deaths were due to ILD and pulmonary artery hypertension(PAH), and the overall in-hospital mortality rate was $16.5 \%$.

Conclusions: Women with SSc had higher rates of hospitalization but lower in-hospital mortality than men.There were some differences between our study and other similar studies in the causes of hospitalization and in-hospital death among SSc patients, especially the lower age of death. The patients with digital ulcers and those with intestinal lung disease or pulmonary hipertension were most commonly admitted to the hospital in our study group. Probably, increasing the skin care of these patients and asking other specialty groups to cooperate will decrease the high rate of hospitalizations in our population.
\end{abstract}

Key words: systemic sclerosis, hospitalization, mortality, length of stay.

\section{Introduction}

Systemic sclerosis (SSc) is an uncommon multisystem disease [1]. It affects about $0.01 \%$ of the population, predominantly women. The disease can occur at any age, but it is most common among middle-aged women [2]. Several advancements in the management of SSc have occurred that may affect the hospitalization pattern [3]. The prognosis for diffuse systemic sclerosis (dcSSc) is worse than limited systemic sclerosis (IcSSc) because of the severe internal organ involvement in the former, especially in the early course of the disease [4].

A large scale study between 2002-2003 evaluated the SSc patients in USA hospitals and the reasons for hospitalization and in-hospital death showed that improvements in the care of patients with SSc in the past several years have had a positive effect on hospitalization patterns. Women had higher hospitalization rates because of the difference in prevalence between genders. Also, they showed that hospitalization rates increased with age after 65 yrs. The most common causes of death were diseases of the circulatory and respiratory systems. The presence of pulmonary fibrosis/interstitial lung disease (ILD) was associated with 2.6 times increased odds of in-hospital death and LOS.The most common reasons for hospitalization were diseases of the circulatory system [i.e. congestive heart failure (CHF), acute myocardial infarction (MI), dysrhythmias] followed 
by diseases of the gastrointestinal, musculoskeletal and respiratory systems, respectively [3].

Another study on 658 admissions of patients with scleroderma in the Hospital of the University of Pennsylvania showed that in-hospital mortality was significantly higher in patients with aspiration events [5].

In a recent study done by Rubio-Rivas from 1990-2009 on Spanish populations, it was shown that SSc-related causes of death are decreasing over time and, among them, pulmonary involvement was still the leading cause of death in both decades. The ratio of renal causes decreased since 1990 at the time that the ratio of cardiac causes increased [6]. Systemic sclerosis with its secondary severe complications is also one of the causes of intensive care unit (ICU) admission $[7,8]$. Given the advances in management of rheumatologic diseases and new guidelines in the last decade, we determined the prevalence of SSc in the last 13 years in our rheumatologic hospitals as a referral center for the southern Iranian patients, the causes of hospitalization, the average length of stay (LOS), and prevalence of SSc hospitalization patterns in more recent years compared to the last 10 years' data; we also evaluated the demographic data of patients during 1999-2012. Also, the mortality rate and the reason for their mortality were studied.

\section{Material and methods}

A cross-sectional study was performed at Hafez (Special Rheumatology hospital), Faghihi (General Hospital including rheumatology ward) and Namazi (General Hospital) hospitals, affiliated to Shiraz University of Medical Sciences, Iran. The studied population included all patients diagnosed with SSC who fulfilled the criteria of disease based on the LeRoy criteria [9]. They were admitted in 3 hospitals of Hafez, Faghihi, and Namazi from April 1999 to May 2012. We excluded all patients with localized scleroderma and also those with mixed connective tissue disease or overlapping syndrome. Principal diagnosis (the primary reason for hospitalization), the other diagnoses in discharge sheet, discharge state including death or otherwise, and the reason for death were included.

Screening, demographic measurements, and clinical assessment were performed by the rheumatologist involved in this research protocol and a general practitioner who was experienced in the assessment of SSc patients.

For better evaluation, the patients were categorized into 7 age groups: < 10, 10-20, 20-30, 30-40, 40-50, 5060 , and $>60$ year-old). Data collection for this study was done using a structured questionnaire. Patients were categorized as having the diffuse or limited disease and demographic information including age, gender, birth place, and living place were recorded.
The other main topics of the questionnaire were the number of admissions for each patient, disease duration before hospitalization, chief complaint (the reason for each admission), principal diagnosis on the discharge sheet, scleroderma-related complications for each patient (i.e. digital tip ulceration, digital gangrene, proximal muscle weakness, severe Raynaud's, PAH, pericardial effusion, ILD, renal failure, hypertension, infections, cardiac disease, cancer, operation, gastric or duodenal ulcer, cerebral or mental disorder), average LOS at hospital, and cause of in-hospital death.

The presence of lung disease was assessed according to their chest radiographs and high resolution computed tomography $(\mathrm{HRCT})$ at the first screening or after detecting low DLCO $<80 \%$ or low FEV $1<75 \%$ in PFT documented by a pulmonologist in their past medical histories or their OPD documents under follow-up of their rheumatologists or as a new detection during their hospital courses. PAH was defined as PAH diagnosed by a cardiologist or right heart catheterization evidence of PAH.

Renal involvement was defined as elevated creatinine and development of renal crisis (hypertension with microangiopathic hemolysis and raised creatinine levels). Cardiac involvement was assessed by electrocardiogram and echocardiography. The presence of other internal organ pathology was obtained from medical records.

For the first step, the patients diagnosed with systemic sclerosis were found and their medical records collected from the hospital files. For the second step, the collected documents were reviewed by the supervisor rheumatologist to confirm the diagnosis of systemic sclerosis for the studied patients, and the patients with an irrelevant diagnosis or incomplete documents were excluded. Then, the documents were read carefully in order to extract the necessary data and fill out the prepared questionnaire.

The data were analyzed in Statistical Package for the Social Sciences (SPSS), version 16, using descriptive statistics. Analytical testing was done to determine if disease characteristics differed by sex and SSc type. Fisher's exact test was used to detect whether there were any possible differences. T-test and CHISQ test were used to compare the variables in the questionnaires. A $p$-value $<0.05$ was considered as a significant difference in this study.

\section{Results}

The analysis included 524 hospitalization cases by 215 patients during 13 years in the main referral hospitals of Shiraz. Seventy-eight cases were excluded, mostly overlapping syndromes. Then, 446 admissions by 181 
patients with systemic sclerosis were considered for further analyses.

There were 408 (91.47\%) female and 38 (8.53\%) male patients. The female to male ratio was about $10.7: 1$. The mean age of the patients was 41.38 , with a minimum of 9 years and maximum of 79 years. The majority of the patients (406) had dcSSc (91.03\%) and 40 (8.97\%) had IcSSc. In another study done in our in- and out-patient's clinics including 533 patients, the female to male ratio was $7.3: 1$. And the ratio of DcSSc and LcSSc was the same. Thus, it seems that the admissions were more frequent in female patients compared to general population of patients with SSc and significantly more in patients with DcSSc [10].

The mean number of admissions was 2.71 times (median 2 times) for each patient (Minimum 1 time and maximum 17 times). During our study period, $54.6 \%$ of the patients just had one hospital admission, $24.3 \%$ had 2 times admissions, $12.1 \%$ were admitted for the $3^{\text {rd }}$ time, $8.2 \%$ for the $4^{\text {th }}$ time and $0.8 \%$ had more than four admissions. The mean disease duration in the studied patients was 7.08 years (minimum 00: new case, and maximum 30 years). The overall mean LOS was 5.95 days (median 5 days) (minimum 1 day and maximum 90 days).

The main scleroderma sign and symptoms detected during hospital course are shown in Table I.

During the hospital course, cardiac diseases were recorded in some of our study patients, i.e. 31 (5.9\%) [including coronary artery disease (CAD) myocardial infarction (MI) and congestive heart failure (CHF)].

Other complications were electrolyte imbalance $33(7.4 \%)$, genitourinary (GU) problem 6 (1.3\%), liver disease 25 (5.6\%), and cerebral and mental disorder 15 (3.4\%). Pancytopenia was detected in 2 (0.4\%) patients in the hospital course. The operation was done on $12(2.7 \%)$ of them during their hospital stay. The most common operation was amputation due to digital gangrene in 7 patients.Other surgeries were cholecystectomy in 2 patients, hemorrhoidectomy in 1 patient, elbow abscess drainage in 1 patient, and laparotomy due to the acute abdomen in 1 patient. Malignancy was detected in $3(0.67 \%)$ patients (lung cancer in 2 patients that were female and ovarian cancer in 1 patient).

The reasons for hospitalization were in 2 categories: SSc-related and non-SSc-related events (Table II).

Digital ulcers were the most common causes of hospitalization among the SSc-related events. For those with a non-SSc-related condition, infection was the most prevalent event.

Fifteen patients referred to the hospital with a complaint of fever (3.4\%) that led to a final diagnosis of 11 patients with pneumonia, 2 patients with pyelonephritis, and 2 patients with exudative tonsillitis.
Table I. The patients' signs and symptoms at each hospital admission

\begin{tabular}{|lc|}
\hline $\begin{array}{l}\text { Common Presenting signs and } \\
\text { symptoms }\end{array}$ & Number (\%) \\
\hline Digital ulcer & $278(62.3)$ \\
\hline Digital gangrene & $54(12.1)$ \\
\hline Digital infection & $28(5.8)$ \\
\hline Pulmonary hypertension & $62(13.9)$ \\
\hline $\begin{array}{l}\text { Interstitial lung } \\
\text { disease }\end{array}$ & $197(44.2)$ \\
\hline Renal failure & $51(11.4)$ \\
\hline Hypertension & $93(20.3)$ \\
\hline Pericardial effusion & $46(10.3)$ \\
\hline Proximal muscle weakness & $75(16.8)$ \\
\hline
\end{tabular}

Table II. Reasons of hospitalization

\begin{tabular}{|c|c|c|c|}
\hline Chief complaint & Frequency & Percent & $\begin{array}{c}\text { Valid } \\
\text { Percent }\end{array}$ \\
\hline \multicolumn{4}{|l|}{ 1. SSc-related events } \\
\hline Digital ulcer & 177 & 39.7 & 39.7 \\
\hline $\begin{array}{l}\text { Scleroderma renal crisis } \\
(\mathrm{SRC})\end{array}$ & 7 & 1.6 & 1.6 \\
\hline Dyspnea/ILD/PAH & 94 & 21.1 & 21.1 \\
\hline Body pain & 36 & 8.1 & 8.1 \\
\hline $\begin{array}{l}\text { Proximal muscle } \\
\text { weakness }\end{array}$ & 19 & 4.3 & 4.3 \\
\hline Digital infection & 23 & 5.2 & 5.2 \\
\hline Digital gangrene & 24 & 5.4 & 5.4 \\
\hline Dysphagia & 6 & 1.3 & 1.3 \\
\hline Arrhythmia & 1 & 0.2 & 0.2 \\
\hline \multicolumn{4}{|l|}{ 2. Non-SSc related events } \\
\hline Fever & 15 & 3.4 & 3.4 \\
\hline Generalized edema & 10 & 2.3 & 2.3 \\
\hline Diarrhea & 12 & 2.7 & 2.7 \\
\hline Urine frequency/urgency & 3 & 0.7 & 0.7 \\
\hline Chest pain & 4 & 0.9 & 0.9 \\
\hline For operation & 1 & 0.2 & 0.2 \\
\hline Rectal bleeding & 2 & 0.4 & 0.4 \\
\hline Bloody vomiting & 3 & 0.7 & 0.7 \\
\hline Jaundice(viral hepatitis) & 2 & 4 & 4 \\
\hline $\begin{array}{l}\text { Abdominal pain } \\
\text { One with Non-ulcer dys- } \\
\text { pepsia and one with ascites } \\
\text { due to ovarian cancer) }\end{array}$ & 2 & 4 & 4 \\
\hline Megaloblastic anemia & 1 & 0.2 & 0.2 \\
\hline Iron deficiency anemia & 3 & 0.7 & 0.7 \\
\hline Increased ESR & 1 & 0.2 & 0.2 \\
\hline
\end{tabular}


Table III. Causes of death and gender distribution of patients with scleroderma who died when hospitalized

\begin{tabular}{|lllc|}
\hline \multicolumn{1}{c}{ Causes of deaths } & \multicolumn{2}{c}{ Gender } & \multirow{2}{*}{$\begin{array}{c}\text { Total } \\
\text { (percent) }\end{array}$} \\
\cline { 2 - 3 } & Female & male & \\
\hline $\begin{array}{l}\text { Systemic sclerosis-related events } \\
\text { ILD/and or PAH }\end{array}$ & 6 & 4 & $10(33.3 \%)$ \\
\hline Scleroderma renal crisis & 1 & 0 & $1(3.3 \%)$ \\
\hline $\begin{array}{l}\text { Cardiac problem } \\
\text { (CHF, MI, arrhythmia) }\end{array}$ & 7 & 1 & $8(26.6 \%)$ \\
\hline $\begin{array}{l}\text { Non-systemic sclerosis-related } \\
\text { events } \\
\text { Cerebrovascular accident (CVA) }\end{array}$ & 4 & 1 & $5(16.6 \%)$ \\
\hline $\begin{array}{l}\text { Massive pulmonary embolism } \\
\text { Sepsis + DIC }\end{array}$ & 1 & 1 & $2(6.6 \%)$ \\
\hline $\begin{array}{l}\text { Febrile neutropenia } \\
\text { with lung metastasis) }\end{array}$ & 1 & 0 & $1(3.3 \%)$ \\
\hline Gl bleeding + couagulopathy & 1 & 0 & $1(3.3 \%)$ \\
\hline
\end{tabular}

During the study period, 30 patients died. Twenty-three patients were females and 7 were males (Table III). The mean age of the patients who died was 43 years (minimum 21 years and maximum 78 years). Diseases of the respiratory and circulatory system were identified as the principal diagnosis in patients who died in the hospital. The mean length of hospital stay in these patients was 7.23 days and the mean duration of the disease was 5.5 years.

Most deaths among the studied patients were due to ILD and PAH (33.3\%) rather than to SRC (3.3\%). Mortality due to scleroderma-related cardiac disease, especially congestive heart failure and arrhythmia, was also common (26.6\%). However, the most prevalent causes of death that were not directly related to SSc were cerebrovascular accident CVA (16.6\%), gastrointestinal (GI) bleeding with coagulopathy (3.3\%), pulmonary thromboembolism (PTE) (6.6\%), sepsis with DIC(3.3\%), febrile neutropenia (3.3\%), and ovarian cancer with metastasis to the lung (3.3\%) (Table III).

\section{Discussion}

This is a study in our center as a referral center for rheumatologic diseases in Shiraz to evaluate hospitalization and mortality of patients with scleroderma during 13 years; we found some differences between our study and the other similar studies as the causes of hospitalization and in-hospital death among SSc patients.

Our patients admitted with SSc were more commonly female (91.4\%) and female to male ratio was about
10.7 : 1 that was higher than most other studies. Chung et al. [3] evaluated in-patients' sample of SSc patients in USA hospitals from 2002 to 2003, reporting that female to male ratio was $4.5: 1$. Also, the female to male ratio was 1.6 : 1 in Sittichai Netwijitpan's study on hospitalized patients [4]. In a study done by Wielosz et al. [11] on European Caucasian patients, this ratio was $3.1: 1$. Although in a study done in Italy (Sardinia) on 4981 hospitalizations in 2001-2012, the women's admissions was $84.8 \%$ that was nearly the same as our patients [12].

In our study, the majority of the patients had dcSSc (91.03\%). Netwijitpan et al. [4] also reported that $81.7 \%$ of the hospitalized SSc patients had dcSSc although in a European study the admissions were more in patients with LCSSc [11].

In presented study, the over all mean LOS was 5.95 days (minimum 1 day and maximum 90 days). Men stayed at hospital more than female patients (6.28 days vs. 5.92 days). Chung et al. [3] found that the overall mean length of stay was 6.6. Netwijitpan et al. [4] reported that mean LOS was 5 days. This result was the same as those of our study.

Our study showed that patients $>60$ years had longer mean LOS than other age groups (8.4 days), and patients < 10 years had shorter mean LOS (2 days). But there was no significant difference between the age group of $>60$ years and other age groups ( $<60$ years). Like previous studies, we found that hospitalization patterns of SSc patients differed by gender and age.Women had higher hospitalization rates than men (2.75 times vs. 2.21 times).

Most of the patients had undergone one hospital admission (54.6\%) and $24.3 \%$ of them had 2 times admissions. $12.1 \%$ of patients were admitted for the $3^{\text {rd }}$ time, $8.2 \%$ for the $4^{\text {th }}$ time and $0.8 \%$ had more than four admissions. Netwijitpan et al. [4] also reported that $63.4 \%$ of SSc patients had one admission, $25.2 \%$ had two, $7.6 \%$ had three, $2.3 \%$ had four, and $1.5 \%$ had more than four admissions.

The most common chief complaint in those 446 hospitalization cases was SSc-related events (i.e. digital ulcer and dyspnea due to ILD/PAH). For those with a non-SSc-related conditions, infection was the most prevalent events. Netwijitpan et al. [4] showed that PAH was the most common cause of hospitalization among the SSc-related events, while for those with a non-SSc-related condition, infection (i.e. pneumonia, diarrhea, and urinary tract infection) was the most common event, so it was the same as our study in admission causes.

Chung et al. [3] found that diseases of the circulatory system [i.e. CHF, acute myocardial infarction (MI), dysrhythmias] were the principal cause of hospitalization in SSc patients (21.9\%), followed by diseases of the 
gastrointestinal (12.7\%), musculoskeletal (12.0\%) and respiratory (11.5\%) systems; $\mathrm{PAH}$ and infection were the $14^{\text {th }}$ and $8^{\text {th }}$ most common diagnoses. In the study by Piga et al. [12] ILD was the most common scleroderma-related causes of admissions and infection was the most common non-scleroderma cause. Their second scleroderma-related cause was procedures(intravenous treatments) that in our patients with digital ulcers was also the main cause of admissions for receiving prostaglandins and we included them as digital ulcer causes. So, maybe the main causes in our patients were relatively the same [12].

Therefore, it seems we should consider digital ulcers as the main hospitalization causes of our patients although most of the patients with digital ulcers were admitted only for receiving intravenous medications and maybe we should be more concerned about this complication and may be day-clinics with more facilities for intravenous treatments available in all of our referral centers can resolve this problem for our patients. The most common non-scleroderma related cause of hospitalization was an infection, like other studies, that should be considered as the main cause of morbidity in our patients.

During our study period, 30 in patients (7\%) died, all of whom had diffuse systemic sclerosis. In another study done by Sehra et al. [5], their mortality in 593 SSc all cause admissions were reported in 30 patients (5\%). We found that in-hospital mortality rate was increased in men more than women. That was the same as Sehara et al. [5] study. The mean age of our patients who died was 43 years that was lower than their study that was 61 years old. Also in the study by Ooi et al. [13], the median age at death was 71 years (range 42-87) that was significantly higher than our patients again. The mean length of hospital stay in these patients was 7.23 days and the mean duration of the disease was 5.5 years. Jacobsen et al. [14] reported that the disease duration did not differ significantly between surviving and deceased patients. But our study showed that disease duration was different among these groups (7.08 years vs. 5.5 years). This was due to the difference in the number of dcSSc and IcSSc who died in our study and Jacobsen et al.'s study. In Jacobsen et al.'s study, $66 \%$ of mortality cases occurred in patients with IcSSc and 34\% in dcSSc. In presented data we found that, all in-hospital mortality cases occurred in dcSSc patients (100\%) [14].

Among studied patients 30 died and hospital mortality rate was $16.5 \%$. Netwijitpan et al. [4] reported that SSc patient's in-hospital mortality rate was 6.3\%; also, Chung found that it was $16.8 \%$ [3].

Most deaths among our patients were due to SSc-related events, ILD, pulmonary HTN (33.3\%) and cardiac problems rather than to scleroderma renal crisis (SRC) (3.3\%). Mortality due to scleroderma-related cardiac disease, especially congestive heart failure and arrhythmia, was also common (26.6\%). However, the most prevalent cause of death not directly related to SSc was cerebrovascular accident (CVA) and pulmonary thromboemboli (PTE) and very rarely malignancy and other causes.

In the study by Ooi et al. [13], the most common leading causes of death were also scleroderma-related causes including PAH and ILD and then SRC.

Netwijitpan et al. [4] reported that non-systemic sclerosis-related events (68.2\%) were the most common causes of in-hospital death (Bacterial pneumonia in $73.2 \%$, pulmonary tuberculosis in $6.7 \%$, H1N1 pneumonitis in 6.7\%, Bronchoalveolar cancer in 6.7\%, and massive pulmonary embolism in $6.7 \%$ ), and systemic sclerosis-related events accounted for $31.8 \%$ mostly due to congestive heart failure in $57.1 \%$ and then intestinal pseudo-obstruction in $14.3 \%$, scleroderma renal crisis in $14.3 \%$, and severe malnutrition in $14.3 \%$.

Several mortality studies in SSc have been performed in the USA and other countries over the past several decades. In these studies, SSc-related internal organ involvement (including renal failure, $\mathrm{PAH}$, pulmonary fibrosis and cachexia/oesophageal dysfunction) as well as ischemic heart disease, CHF, infections and malignancies were the most common causes of death. As indicated in Chung et al.'s [3] study, SSc-related events were the most common cause of death in our patients.

Our analysis showed that $3.3 \%$ of deaths were due to malignancy (only one case, ovarian cancer with metastasis to the lung). Chung et al. [3] found that malignancy was the cause of deaths in $5.76 \%$ of SSc patients who died in the hospital. Netwijitpan et al. [4] showed that $6.7 \%$ of in-hospital mortality deaths were due to bronchoalveolar cancer.

We recommend further studies on main malignancies in our patients with scleroderma and maybe the pattern of malignancies is different in our patients. Also, we did not know about the whole causes of mortality including outpatient causes. Therefore, future studies can help us to define the main causes of death in our population.

\section{Conclusions}

Women with SSc had higher rates of hospitalization but lower in-hospital mortality than men. There were some differences between our study and other similar studies in the causes of hospitalization and in-hospital death among SSc patients, especially the lower age of death. The patients with digital ulcers admitted for in- 
travenous treatments and then ILD/PAH were the main cause of hospitalization in our patients.

The present article was extracted from the thesis written by Dr. Razieh Naseri and financially supported by Shiraz University of Medical Science Grant number: 904066. The authors would like to thank Shiraz University of Medical Sciences, Shiraz, Iran and also Center for Development of Clinical Research of Namazi Hospital and Dr. Nasrin Shokrpour for editorial assistance.

The authors declare no conflict of interest.

\section{References}

1. Medical Grand Rounds from the University of Alabama Medical Center. Lupus erythematosus. South Med J 1971; 64: 839846.

2. Yazawa N, Fujimoto M, Tamaki K. Recent advances on pathogenesis and therapies in systemic sclerosis. Clin Rev Allergy Immunol 2007; 33: 107-112.

3. Chung L, Krishnan E, Chakravarty EF. Hospitalizations and mortality in systemic sclerosis: results from the Nationwide Inpatient Sample. Rheumatology (Oxford, England) 2007; 46: 1808-1813.

4. Netwijitpan S, Foocharoen C, Mahakkanukrauh A, et al. Indications for hospitalization and in-hospital mortality in Thai systemic sclerosis. Clin Rheum 2013; 32: 361-367.

5. Sehra ST, Kelly A, Baker JF, Derk CT. Predictors of inpatient mortality in patients with systemic sclerosis: a case control study. Clin Rheum 2016; 35: 1631-1635.

6. Rubio-Rivas M, Simeon-Aznar CP, Velasco C, et al. Changes in the pattern of death of 987 patients with systemic sclerosis from 1990 to 2009 from the nationwide Spanish Scleroderma Registry (RESCLE). Clin Exp Rheumatol 2017.

7. Shalev T, Haviv Y, Segal E, et al. Outcome of patients with scleroderma admitted to intensive care unit. A report of nine cases. Clin Exp Rheumatol 2006; 24: 380-386.

8. Heijnen T, Wilmer A, Blockmans D, Henckaerts L. Outcome of patients with systemic diseases admitted to the medical intensive care unit of a tertiary referral hospital: a single-centre retrospective study. Scand I Rheum 2016; 45: 146-150.

9. LeRoy EC, Black C, Fleischmajer R, et al. Scleroderma (systemic sclerosis): classification, subsets and pathogenesis. J Rheumatol 1988; 15: 202-205.

10. Nazarinia MA, Esmaeilzadeh E, Emami Y, Salehi A. One decade distinct features, morbidity and mortality of scleroderma: a cross-sectional study. Clin Exp Rheumatol 2016; 34 Suppl 100 (5): 74-78.

11. Wielosz E, Majdan M, Dryglewska M, Suszek D. Comparison of clinical and serological parameters in female and male patients with systemic sclerosis. Reumatologia 2015; 53: 315320.

12. Piga M, Casula L, Sanna S, et al. Population-based analysis of hospitalizations for patients with systemic sclerosis in a West-European region over the period 2001-2012. Rheum Intern 2016; 36: 73-81.
13. Ooi C, Solanki K. Mortality in the Waikato Hospital Systemic Sclerosis Cohort. Int J Rheum Dis 2017; doi: 10.1111/1756185X.13111.

14. Jacobsen S, Halberg P, Ullman S. Mortality and causes of death of 344 Danish patients with systemic sclerosis (scleroderma). Br J Rheum 1998; 37: 750-755. 